\title{
Nicotinamide induces liver regeneration and improves liver function by activating SIRT1
}

\author{
HAI-FENG WAN $^{1 *}$, JIA-XIN LI $^{1 *}$, HAO-TIAN LIAO ${ }^{1 *}$, MING-HENG LIAO $^{1}$, \\ LIN LUO $^{1}$, LIN XU ${ }^{2}$, KE-FEI YUAN ${ }^{1}$ and YONG ZENG ${ }^{1}$ \\ ${ }^{1}$ Department of Liver Surgery, Liver Transplantation Division; ${ }^{2}$ Laboratory of Liver Surgery, \\ West China Hospital, Sichuan University, Chengdu, Sichuan 610041, P.R. China
}

Received December 12, 2017; Accepted September 21, 2018

DOI: $10.3892 / \mathrm{mmr} .2018 .9688$

\begin{abstract}
Nicotinamide (Nam) has recently been characterized as an agent for tissue regeneration due to the observed pro-proliferation effects. However, the effect of Nam on liver regeneration remains undetermined. In the present study, the potency of Nam as a regimen to promote liver regeneration and restore liver function was evaluated following partial hepatectomy (PH) on C57BL/6 mice. Ki-67 immunohistochemical and cell cycle analyses demonstrated that exogenous Nam supplementation promoted the proliferation of hepatocytes and accelerated the recovery of liver tissue. The addition of Nam protected liver function following $\mathrm{PH}$, as evidenced by hematoxylin and eosin staining of liver tissue morphology and measurement of serum liver injury markers. Notably, immunoblotting results revealed that the expression and activity of NAD-dependent protein deacetylase sirtuin-1 (SIRT1) were significantly upregulated following treatment with Nam, suggesting that Nam may promote liver regeneration through activation of SIRT1. The present study demonstrated that Nam regulated the process of liver regeneration and improved liver function by activating SIRT1, suggesting that Nam has the potency to be used for promoting liver regeneration following surgical resection.
\end{abstract}

\section{Introduction}

Hepatocellular carcinoma (HCC) has been ranked the third most life-threatening cancer, causing approximately

Correspondence to: Professor Ke-Fei Yuan or Professor Yong Zeng, Department of Liver Surgery, Liver Transplantation Division, West China Hospital, Sichuan University, 37 Guoxue Street, Chengdu, Sichuan 610041, P.R. China

E-mail: ykf13@163.com

E-mail: zengyong@medmail.com.cn

${ }^{*}$ Contributed equally

Key words: nicotinamide, NAD-dependent protein deacetylase sirtuin-1, liver regeneration
700,000 mortalities each year globally (1). For patients with HCC at Barcelona Clinic Liver Cancer (BCLC) $(2,3)$ stage 0 or A, surgical resection is the first-line treatment, which contributes to $60-80 \%$ of patients surviving for 5 years $(4,5)$. Surgical resection is applicable in patients with HCC as the liver has a unique capacity to regenerate, following principal tissue loss (6). Notably, there are numerous previous studies that have reported that in patients with HCC beyond BCLC stage A, surgical resection was infrequently the best choice and improved the rate of survival $(2,7,8)$. However, in order to remove the lesions thoroughly, extensive liver resection may be required, leaving an insufficient amount of residual tissue for the liver to recover to maintain vital function (9). On this occasion, a transient fatal form of hepatic failure may develop, leading to the untreatable small-for-size syndrome $(9,10)$. Therefore, identifying a strategy to promote liver regeneration and liver function recovery following partial hepatectomy $(\mathrm{PH})$ is urgently required.

Nicotinamide (Nam) is a form of vitamin $B_{3}$ that is absorbed from our daily diets (11). The therapeutic potential of Nam has been demonstrated in a diverse range of diseases, including skin disorders, diabetes and multiple sclerosis (12-14). Previous studies have revealed that Nam may accelerate the repair of human skin following injury, suggesting the potency of Nam in promoting the regeneration of vital tissues $(15,16)$. It was additionally reported that Nam serves as a precursor of oxidized nicotinamide adenine dinucleotide $\left(\mathrm{NAD}^{+}\right)$during the metabolism of hepatocytes in liver tissue (17-20). However, the effect of Nam on liver regeneration remains unknown.

Sirtuins are class III histone deacetylases that consume one molecule of $\mathrm{NAD}^{+}$during each deacetylation cycle (21). Accumulating evidence has demonstrated that NAD-dependent protein deacetylase sirtuin-1 (SIRT1), a member of the Sirtuin family, regulates numerous metabolic processes in the liver, including gluconeogenesis and lipid synthesis, by directly deacetylating target proteins $(22,23)$. Furthermore, it has been demonstrated that downregulation of SIRT1 impairs liver regeneration (24). According to our previous study, the expression of SIRT1 in hepatocytes was significantly upregulated by Nam (25). Collectively, these results suggest that Nam may promote liver regeneration through the SIRT1-mediated pathways. 
In the present study, it was demonstrated that Nam induced the process of liver regeneration and improved liver function through activation of SIRT1, suggesting that Nam has the potency of becoming a regimen for promoting liver regeneration following surgical resection.

\section{Materials and methods}

Animals and treatment. In the present study, male C57BL/6 mice (6-8 weeks old; $15-20 \mathrm{~g}$; $\mathrm{n}=30$, 6 per group; Beijing HFK Bioscience Co., Ltd., Beijing, China) were raised in specific pathogen-free conditions $\left(23 \pm 1^{\circ} \mathrm{C}\right.$; relative humidity, $39-43 \%$; $12 \mathrm{~h}$ light/dark cycle; free access to food and water). Animal care and experimental protocols were conducted in accordance with guidelines provided by the Institutional Animal Care and Use Committee of Sichuan University (Chengdu, China). Ethics approval was obtained from the Institutional Ethics Committee of Sichuan University. Prior to subjecting the mice to $70 \% \mathrm{PH}$, mice were anesthetized with a subcutaneous injection of pentobarbital at a dose of $80 \mathrm{mg} / \mathrm{kg}$. For the study group, Nam ( $250 \mathrm{mg} / \mathrm{kg}$ per day) was administered $6 \mathrm{~h}$ prior to $\mathrm{PH}$ via intraperitoneal injection and once daily subsequently for a total of 3 days. Control animals were treated with the same frequency and volume of PBS as Nam. All of the study mice were sacrificed at the indicated time points $(24,36,48$ and $72 \mathrm{~h}$ ) and the liver samples were harvested and stored in liquid nitrogen. Liver recovery rates were calculated as the ratio of the regenerating liver weight to the mean liver weight prior to $\mathrm{PH}$. The serum samples $(1 \mathrm{ml})$ were additionally collected from the angular veins of the mice. Blood glucose (Glu), triglyceride (TG), aspartate aminotransferase (AST) and alanine aminotransferase (ALT) were measured using enzymatic methods (ADVIA 165; Siemens Healthineers, Erlangen, Germany).

Protein isolation and western blotting. To obtain the total protein, the liver tissues were frozen in liquid nitrogen, washed twice with PBS, and homogenized in radioimmunoprecipitation assay lysis buffer (Pierce; Thermo Fisher Scientific, Inc., Waltham, MA, USA). The samples were incubated on ice for $30 \mathrm{~min}$ prior to centrifugation at $4^{\circ} \mathrm{C}$ for $15 \mathrm{~min}$ at a speed of $12,000 \times \mathrm{g}$. Subsequent to centrifugation, the supernatant was divided into small fractions and stored at $-80^{\circ} \mathrm{C}$. The protein content of each supernatant was quantified using the Bradford assay (Bio-Rad Laboratories, Inc., Hercules, CA, USA), according to the manufacturer's protocol. Western blot analysis was performed as described previously (26). Cyclin D1 (1:1,000; rabbit monoclonal; cat. no. ab134175), PCNA (1:1,000; mouse monoclonal; cat. no. ab29), and SIRT1 (1:1,000; mouse monoclonal; cat. no. ab110304) primary antibodies were obtained from Abcam (Cambridge, UK); $\beta$-actin $(1: 1,000$; mouse monoclonal; cat. no. sc-69879), as well as the secondary antibodies [horseradish peroxidase (HRP)-conjugated goat anti-mouse and goat anti-rabbit; both 1:5,000; cat. no. sc-2005 and sc-2004] were obtained from Santa Cruz Biotechnology, Inc. (Dallas, TX, USA). Densitometric analysis was performed using ImageJ software (version 1.48; National Institutes of health, Bethesda, MD, USA).

Histology and immunohistochemistry. For immunohistochemical analysis and morphological examination [hematoxylin and eosin (H\&E) staining], formalin-fixed (10\% buffered formalin at $4^{\circ} \mathrm{C}$ overnight), paraffin embedded liver tissues were sectioned to $5 \mu \mathrm{m}$. H\&E staining was performed as described previously (27).

For immunohistochemical analysis, sections were blocked with $5 \%$ bovine serum albumin (Biowest, Nuaillé, France) diluted in PBS (pH 7.4) for $1 \mathrm{~h}$ at $37^{\circ} \mathrm{C}$. Proliferation marker protein Ki-67 (Ki-67) staining was performed with an anti-Ki-67 antibody (1:4,000; rabbit polyclonal; cat. no. ab15580; Abcam) at $4^{\circ} \mathrm{C}$ overnight. Next, slides were incubated with the HRP-conjugated goat anti-rabbit antibody at $37^{\circ} \mathrm{C}$ for $1 \mathrm{~h}$. Liver sections examined at each time-point were from at least three individual animals in the treatment and control groups. Images were captured with the AX10 imager A2 fluorescence microscope (magnification, $x 40$; Carl Zeiss AG, Oberkochen, Germany). Percentage Ki-67 expression was evaluated in five random fields from each immunostained HCC section. Quantification of Ki-67 staining was performed using Image-Pro Plus version 6.0 (National Institutes of Health, Bethesda, MD, USA).

Bioinformatics analysis. The Kyoto Encyclopedia of Genes and Genomes database (https://www.kegg.jp) was used to explore the potential genes regulated by SIRT1.

Acetylation assays. To detect the deacetylation of peroxisome proliferator-activated receptor g coactivator 1- $\alpha$ (PGC-1 $\alpha)$ and oxysterols receptor liver X receptor- $\alpha$ (LXR $\alpha)$ by SIRT1, lysine acetylation was analyzed by immunoprecipitation and western blotting, using an acetyl-lysine antibody. Primary antibodies against PGC-1 $\alpha,(1: 1,000$; rabbit polyclonal; cat. no. ab54481) LXR $\alpha$ (1:1,000; mouse monoclonal; cat. no. ab41902) and acetyl-lysine (1:1,000; rabbit polyclonal; cat. no. ab21623) were purchased from Abcam. HRP-conjugated goat anti-mouse and goat anti-rabbit secondary antibodies were used, as described above, and the western blotting procedure was the same as aforementioned (26). An immunoprecipitation assay was performed as previously described $(28,29)$ using the Pierce ${ }^{\mathrm{TM}}$ Classic Immunoprecipitation kit (Thermo Fisher Scientific, Inc.), according to the manufacturer's protocol.

Statistical analysis. Statistical analysis was performed using GraphPad Prism 6 (GraphPad Software, Inc., La Jolla, CA, USA). A Student's t-test was performed to compare continuous values between the treatment and control groups. Results are presented as the mean \pm standard deviation of three independent experiments. $\mathrm{P}<0.05$ was considered to indicate a statistically significant difference.

\section{Results}

Nam promotes liver proliferation following $\mathrm{PH}$. To investigate the effect of Nam on the process of liver regeneration, $70 \% \mathrm{PH}$ was conducted on the treatment and control groups. As demonstrated in Fig. 1A, the Ki-67 immunohistochemistry staining was performed to validate hepatic cell proliferation following PH. It was observed that when compared with the control group, increased active proliferation was observed in the treatment group at 24-72 h post-operation. Furthermore, quantification of the Ki-67 staining revealed a significant restoration of hepatocyte proliferation in the treatment group 
A
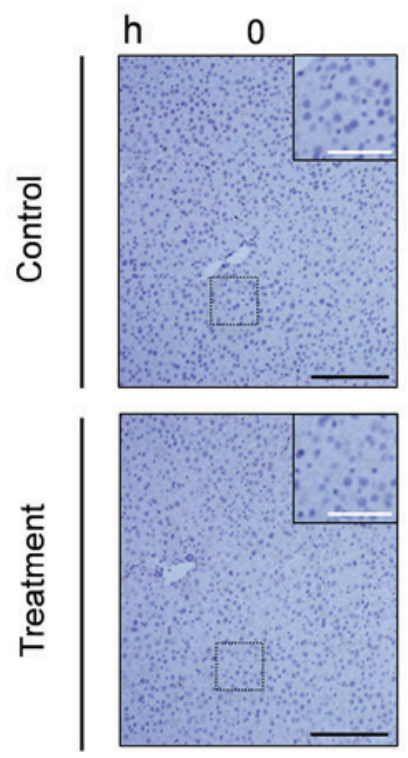

B

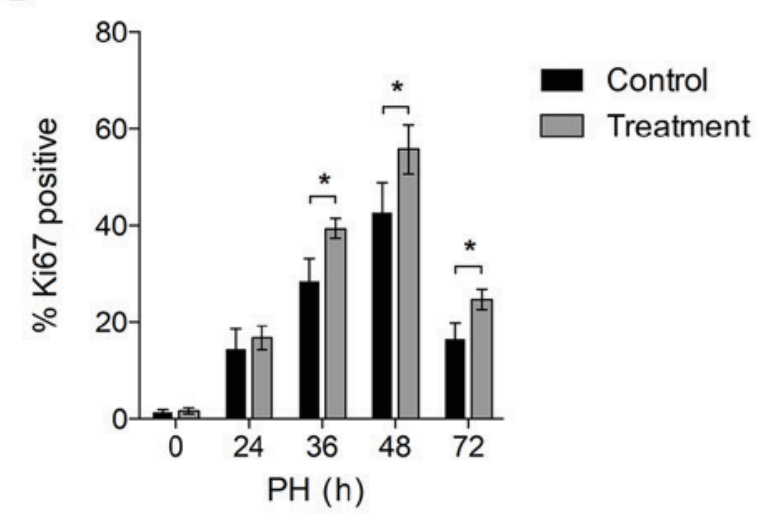

24
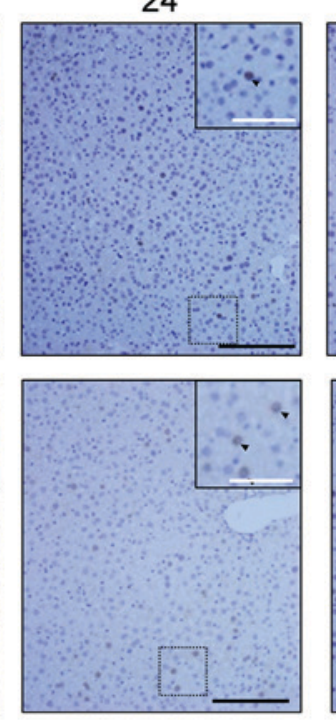

36
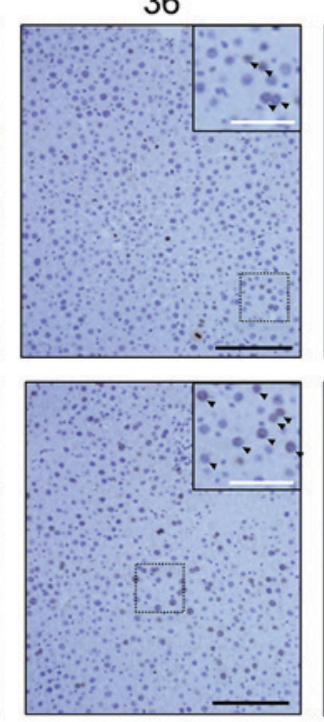

C
48
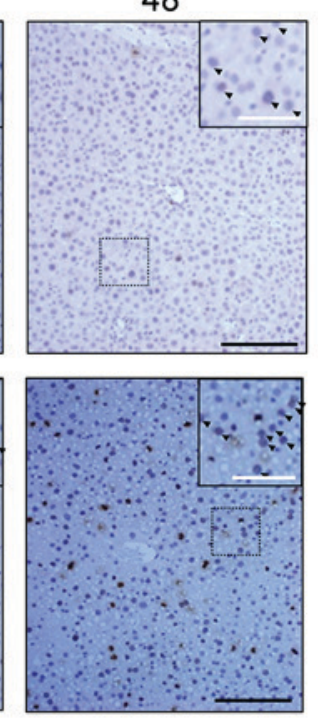

72
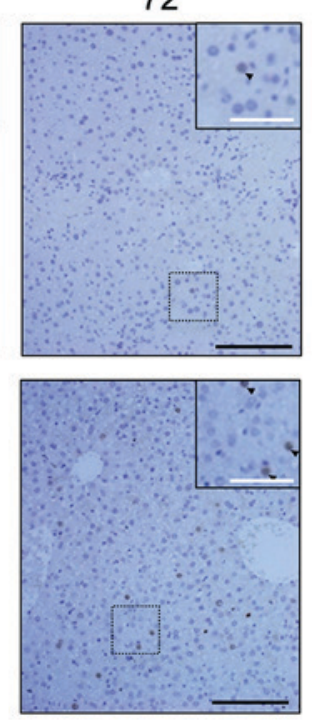

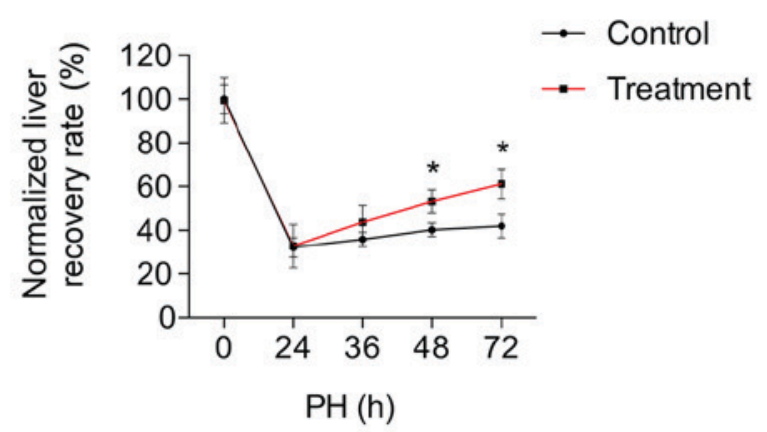

Figure 1. Exogenous supplementation of nicotinamide promotes liver regeneration. (A) Immunohistochemistry for Ki-67 at the indicated time points; the positive cells are marked with arrows. White scale bars, $50 \mu \mathrm{m}$; Black scale bars, $200 \mu \mathrm{m}$. (B) Quantification of Ki-67-positive hepatocytes at the indicated time points. (C) Normalized liver recovery rates are demonstrated as the ratio of the weight of regenerating livers to their mean weight prior to PH. All data are presented as the mean \pm standard deviation $(n=3-5) .{ }^{*} \mathrm{P}<0.05$, as indicated. Ki-67, proliferation marker protein Ki-67; PH, partial hepatectomy.

36-72 h following surgery (Fig. 1B; $\mathrm{P}<0.05$ ). The treatment group additionally exhibited higher liver recovery rates compared with the control group (Fig. 1C). These results suggested that the proliferation of primary hepatocytes was enhanced in the presence of Nam.

Cell cycle progression serves a vital role during liver regeneration (30). To examine the cell cycle of proliferating hepatocytes following $\mathrm{PH}$, the expression of $\mathrm{G}_{1} / \mathrm{S}$-specific cyclin D1 and proliferating cell nuclear antigen (PCNA), two key regulators of the cell cycle, was analyzed by western blotting (Fig. 2A). It was identified that the expression levels of cyclin D1 and PCNA reached a peak at $72 \mathrm{~h}$ in the livers of mice from the treatment and control groups. Additionally, the livers of mice injected with Nam exhibited significantly increased expression of cyclin D1 at 24,36, 48 and $72 \mathrm{~h}$ and PCNA at 0 and $24 \mathrm{~h}$ when compared with the control (Fig. 2B and $C ; P<0.05)$. These results suggested that Nam may accelerate liver regeneration following $\mathrm{PH}$.

Damage caused by PH is repaired in the Nam-treated group. A previous study reported that severe liver damage may occur in short time periods following PH (31). To assess whether Nam may reduce liver damage following $\mathrm{PH}, \mathrm{H} \& \mathrm{E}$ staining was conducted on liver tissue sections. The results of the H\&E staining revealed that the liver tissues of the control group exhibited evident swelling, fatty degeneration and nuclear condensation of hepatocytes $48 \mathrm{~h}$ following $\mathrm{PH}$, along with severe sinusoidal narrowing (Fig. 3A). By contrast, sections from the treatment group demonstrated patent sinusoids, and better preservation of the cytoplasm and nuclear morphology, suggesting that treatment with Nam may repair liver damage following PH.

ALT and AST are markers of liver injury following PH. According to a previous study, these two markers increase markedly following PH (31). To further determine whether Nam has the potential to reduce liver injury following PH, the serum content of ALT and AST was analyzed (Fig. 3B and C). It was observed that the serum levels of ALT and AST were significantly decreased following $\mathrm{PH}$ in the treatment group compared with the control group (Fig. 3B and C; $\mathrm{P}<0.001$ ), suggesting that Nam may protect liver function following trauma caused by $\mathrm{PH}$.

Nam upregulates the expression of SIRT1. Our previous study demonstrated that the addition of Nam promoted the expression 
A
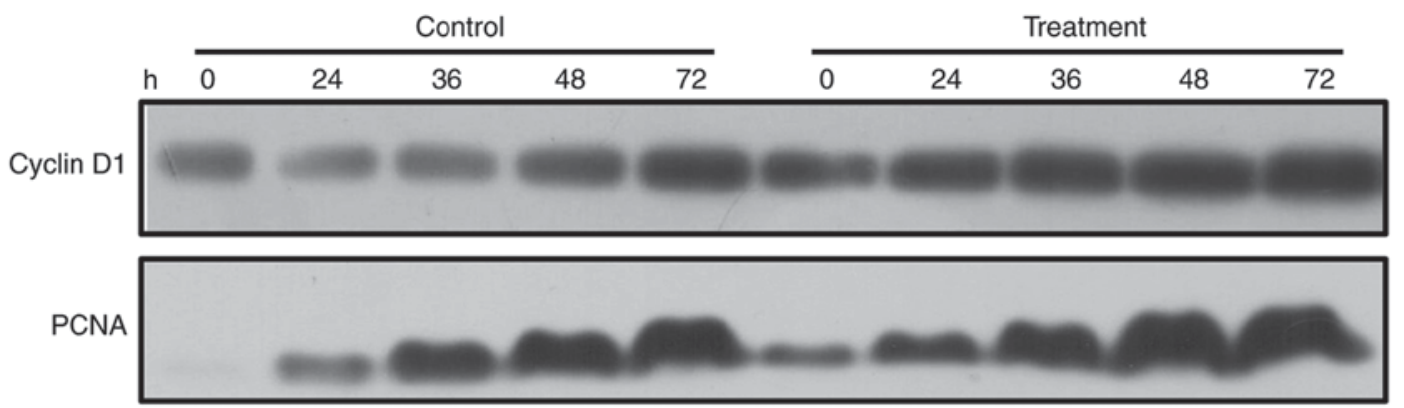

$\beta$-actin
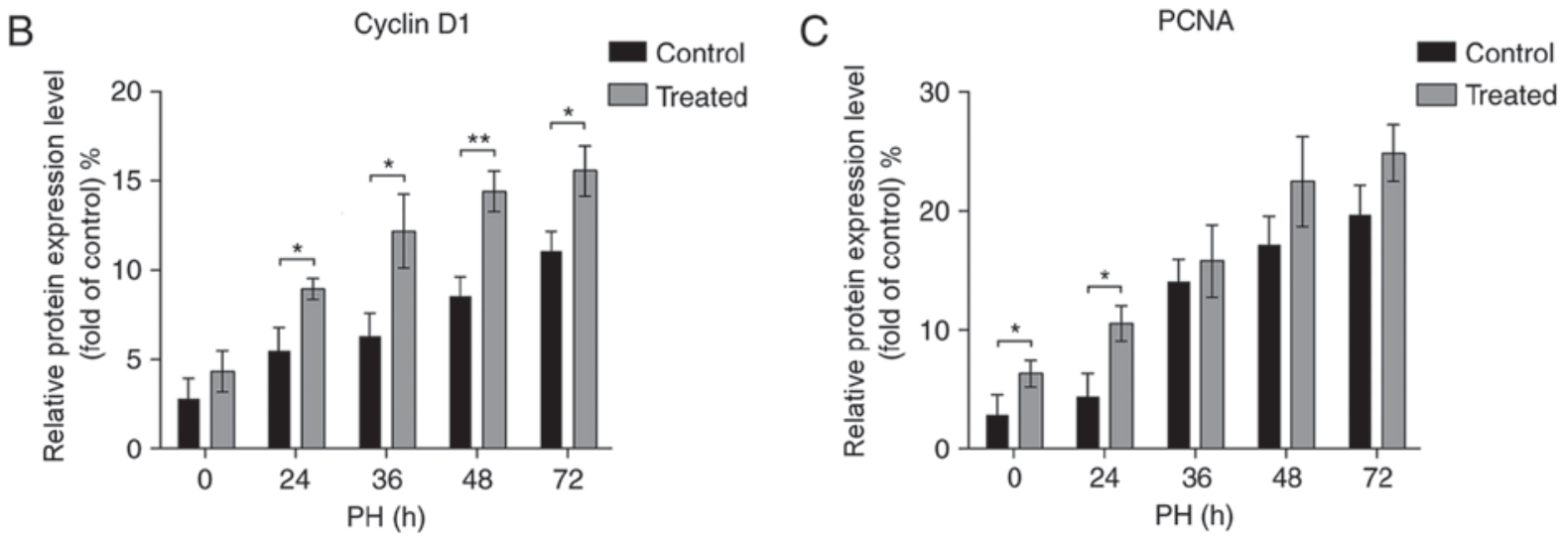

Figure 2. Expression of cyclin D1 and PCNA is increased in mice treated with nicotinamide compared with the control mice. (A) Western blots of the expressions of cyclin D1 and PCNA in the remnant livers following PH. Quantification of the protein expression levels of (B) cyclin D1 and (C) PCNA. Densitometry quantification of the band intensities was conducted using Image J software and is presented as a percentage of relative densitometry normalized to $\beta$-actin. Data are presented as the mean \pm standard deviation of three independent experiments $(\mathrm{n}=3-5)$. ${ }^{*} \mathrm{P}<0.05$ and ${ }^{* *} \mathrm{P}<0.01$, as indicated. Cyclin $\mathrm{D} 1, \mathrm{G}_{1} / \mathrm{S}$-specific cyclin-D1; PCNA, proliferating cell nuclear antigen; $\mathrm{PH}$, partial hepatectomy.

of SIRT1, a key regulator of liver regeneration, suggesting that Nam may promote liver regeneration by upregulating the expression of SIRT1 (25). To verify the mechanisms of Nam in promoting liver regeneration, western blotting analysis was conducted to determine the expression of SIRT1 in the treatment and control groups. As demonstrated in Fig. 4A and B, although the expression of SIRT1 following PH increased in the two groups in a time-dependent manner, the addition of Nam further facilitated the significantly increased expressions of SIRT1 24-72 h following PH (Fig. 4A and B; P<0.05).

Glu and TG metabolisms have been reported to be a prerequisite for liver regeneration $(23,32)$. Previous studies have demonstrated that the levels of Glu and TG are modulated by SIRT1 during the process of liver regeneration $(24,33,34)$. To further elucidate the mechanisms of Nam in promoting liver regeneration, the levels of TG and Glu from serum samples were analyzed. Compared with the control group, the levels of Glu and TG were restored faster in the treatment group (Fig. 4C and D), which was consistent with the result that the expression of SIRT1 was upregulated by Nam. These results suggest that Nam may elevate the levels of Glu and TG by promoting the expression of SIRT1, thereby accelerating the process of liver regeneration.

Deacetylating ability of SIRT1 is enhanced following treatment with Nam. To further investigate the mechanism underlying SIRT1-mediated metabolic processes following treatment with Nam, bioinformatics analysis was conducted (data not shown), which identified two substrates of SIRT1, PGC-1 $\alpha$ and LXR $\alpha$, which may be deacetylated by SIRT1 and have been previously implicated in the process of glucogenesis and lipid synthesis, respectively $(35,36)$. As demonstrated in Fig. 5A and C, compared with the control group, mice injected with Nam demonstrated a significant decrease in the lysine acetylation level of PGC-1 $\alpha(\mathrm{P}<0.01)$, suggesting that Nam may regulate Glu levels through the SIRT1-PGC-1 $\alpha$ signaling pathways. Likewise, it was additionally observed that there was a significant decrease in the lysine acetylation level of LXR $\alpha$ in the treated group (Fig. 5B and D; P<0.01), suggesting that Nam may regulate lipid levels through the SIRT1-LXR $\alpha$ signaling pathways.

\section{Discussion}

At present, one of the clinical concerns regarding liver regeneration is identifying effective regimens to accelerate liver regeneration following surgical resection $(37,38)$. Conventionally, Nam has been regarded as an anti-inflammatory, anti-oxidant and anti-apoptotic agent (39). Previous studies have demonstrated that Nam may promote the expression of SIRT1, an important regulator in liver 
A h
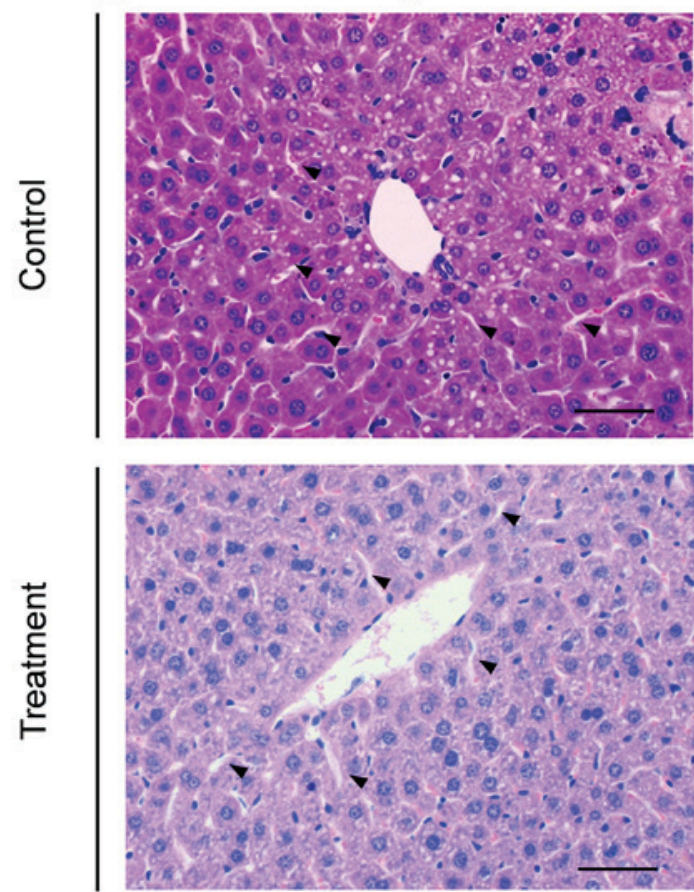

B

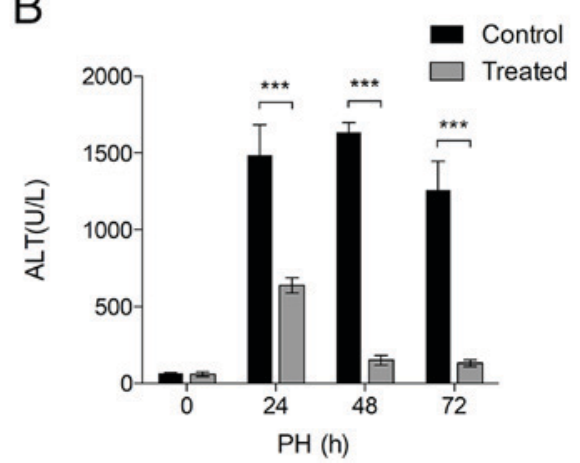

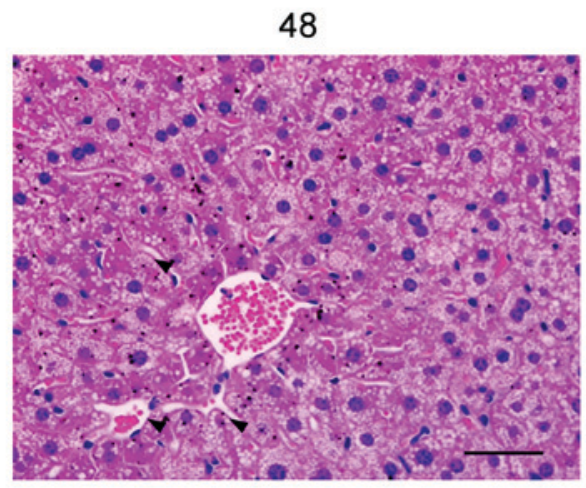

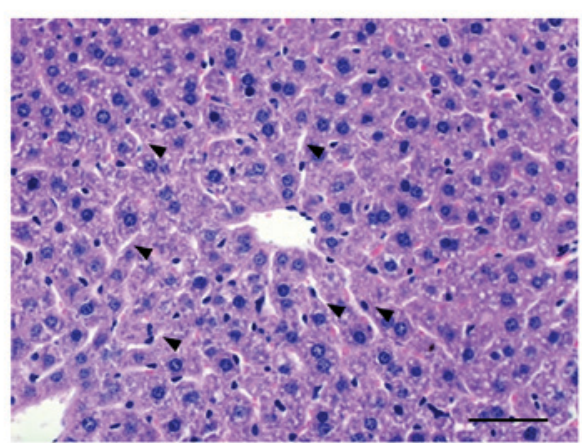

C
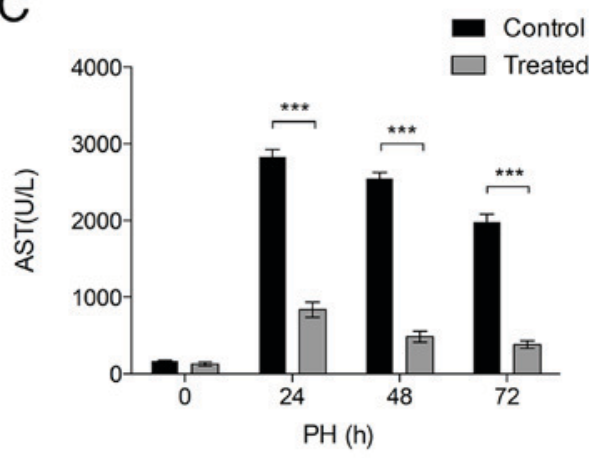

Figure 3. Damage caused by PH is repaired following treatment with Nam. (A) Hematoxylin and eosin staining demonstrated that Nam-treated livers exhibit patent sinusoids (marked with arrows) and less hepatocyte vacuolar alteration. Scale bar, $50 \mu \mathrm{m}$. (B) ALT and (C) AST levels at the indicated time points following PH. All data are presented as the mean \pm standard deviation $(n=3-5) .{ }^{* * *} \mathrm{P}<0.001$, as indicated. PH, partial hepatectomy; Nam, nicotinamide; ALT, alanine aminotransferase; AST, aspartate aminotransferase.

regeneration in hepatocytes $(25,33,34)$, indicating that Nam has the potential to promote liver regeneration. In the present study, the results demonstrated that the addition of Nam promoted hepatocyte proliferation and induced the activation of SIRT1. Furthermore, the present results are supported as Nam may accelerate skin tissue regeneration following physicochemical injuries $(15,16)$, suggesting that Nam has the potential for application in vital tissue regeneration; however, this requires further examination.

Hepatic metabolism, including modulations in serum Glu and lipid, has been revealed to be an essential part of liver regeneration (26). Within hours of surgery, mice subjected to $\mathrm{PH}$ develop significant hypoglycemia when compared with the control group (40). Subsequently, adaptation by modulating the level of blood Glu occurs, including the induction of hepatic gluconeogenic machinery and the suppression of liver glycolytic activity (41). The regulation of lipid is additionally significant for liver regeneration (42). Lipid has always been considered to be a structural component of biological membranes. Furthermore, lipid is involved in the regulation of the intermediate metabolism of different liver cell types, including hepatocytes, hepatic stellate cells and Kupffer cells (42). In the present study, it was demonstrated that Nam promoted the proliferation of hepatocytes, in addition to Glu and lipid metabolism, which was indicative of an association between metabolism and liver regeneration.

SIRT1 has been reported to serve an essential role in various metabolic processes of the liver. During the process of liver regeneration, SIRT1 has been recognized as an important regulator of metabolic circadian rhythms, which have been revealed to be closely associated with cellular proliferation (43-46). Furthermore, it was observed that SIRT1 serves a central role in maintaining proper circadian regulation of metabolic processes, including gluconeogenesis and lipid synthesis, thus affecting the process of liver regeneration $(33,34)$. However, the detailed molecular mechanisms underlying SIRT1-mediated regulation of liver regeneration have not been fully elucidated. In the present 
A
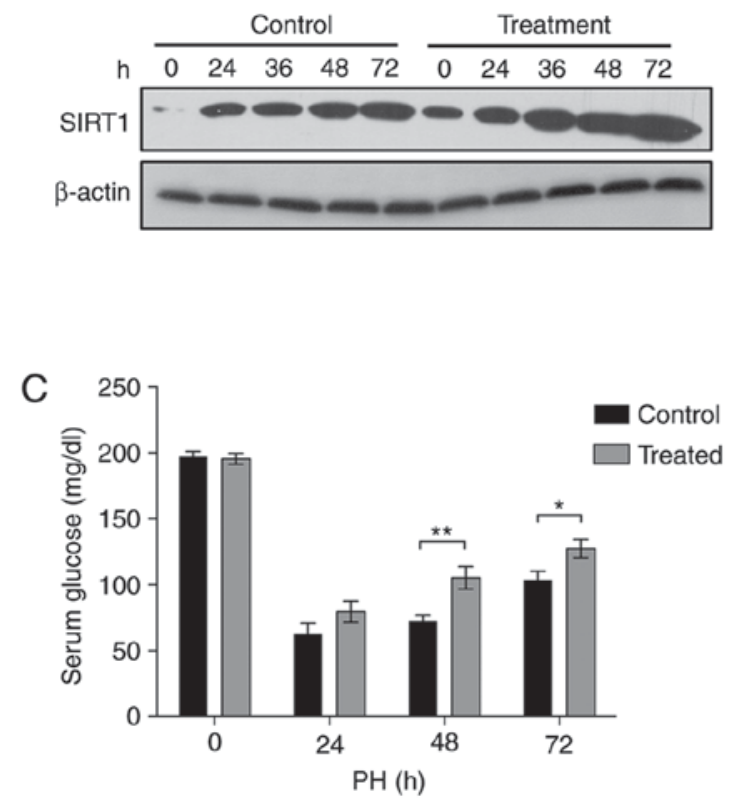
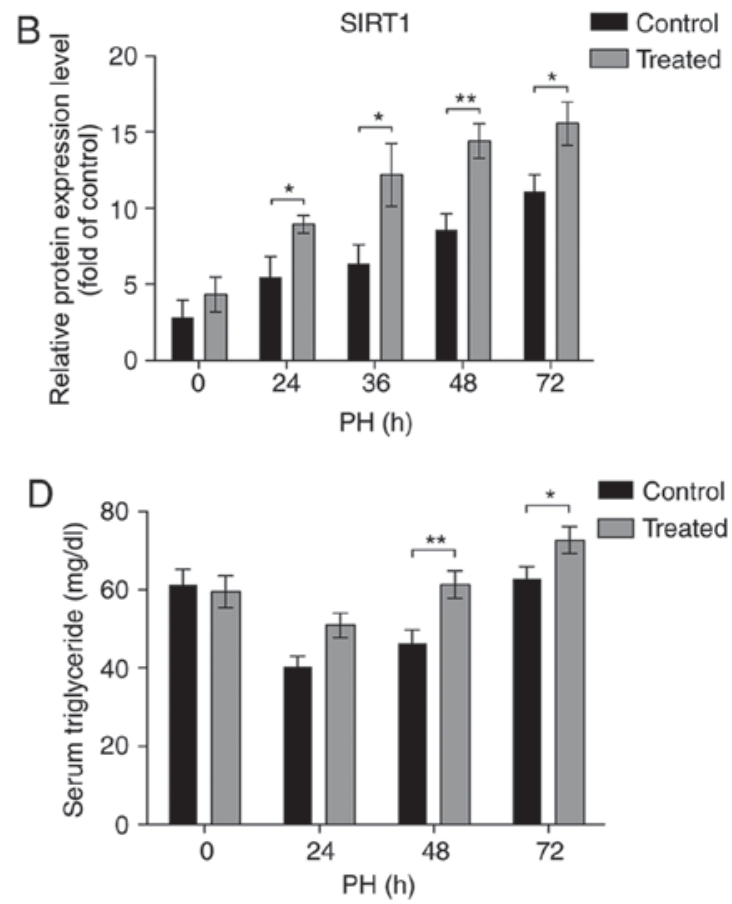

Figure 4. Expression of SIRT1 is higher in mice treated with nicotinamide compared with the control mice treated with PBS. (A) Western blots of the expressions of SIRT1 in the remnant livers following PH. (B) Quantification of SIRT1 protein expression levels. (C) Levels of glucose were determined at the indicated time points following PH. (D) Levels of triglyceride were determined at the indicated time points following PH. All data are presented as the mean \pm standard deviation $(n=3-5)$. $\mathrm{P}<0.05$ and ${ }^{* *} \mathrm{P}<0.01$, as indicated. SIRT1, NAD-dependent protein deacetylase Sirtuin-1; PH, partial hepatectomy.

A

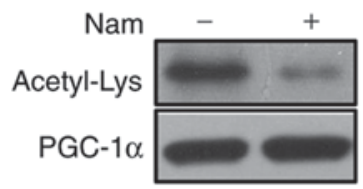

B

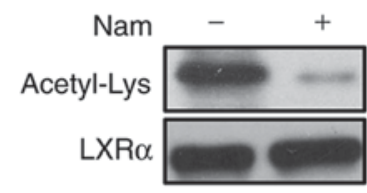

C

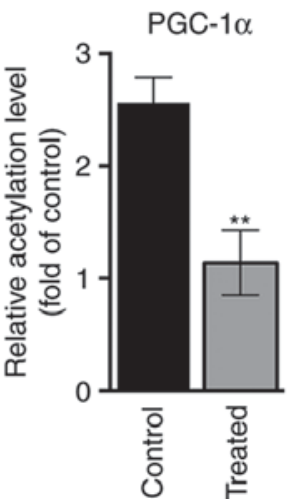

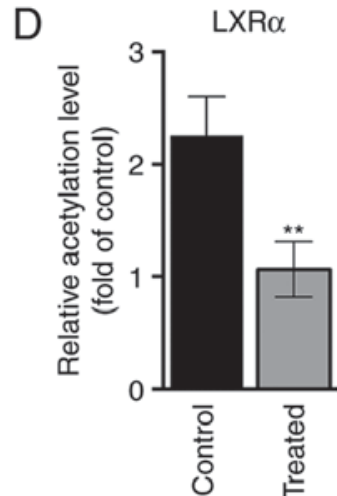

Figure 5. Acetylation levels of PGC-1 $\alpha$ and LXR $\alpha$ decrease in mice treated with Nam. Immunoprecipitation was performed to detect the acetylation of (A) PGC-1 $\alpha$ and (B) LXR $\alpha$. Quantification of (C) PGC-1 $\alpha$ and (D) LXR $\alpha$ protein expression levels. All data are presented as the mean \pm standard deviation $(\mathrm{n}=3-5) .{ }^{* *} \mathrm{P}<0.01$ vs. control. PGC-1 $\alpha$, peroxisome proliferator-activated receptor $\gamma$ coactivator $1-\alpha$; LXR $\alpha$, oxysterols receptor liver X receptor- $\alpha$; Nam, nicotinamide; Acetyl-Lys, acetylated lysine.

study, it was observed that the acetylation levels of PGC-1 $\alpha$ and $\mathrm{LXR} \alpha$ were significantly decreased upon treatment with Nam, suggesting that Nam-induced SIRT1 activation may promote liver regeneration by inducing the deacetylation of PGC- $1 \alpha$ and LXR $\alpha$, thereby promoting gluconeogenesis and lipid synthesis during the process of liver regeneration.

The role of Nam in regulating the activation of SIRT1 remains controversial. Nam is generally accepted as the product of SIRT-catalyzed deacetylation reactions and was reported to inhibit the activity of SIRT1 $(35,36)$. However, as Nam is the predominant precursor for cellular $\mathrm{NAD}^{+}$biosynthesis via the salvage pathway $(47,48)$, a number of previous studies have reported that Nam supplementation in cell culture increased intracellular $\mathrm{NAD}^{+}$levels, leading to the activation of SIRT1 $(49,50)$. Our previous study demonstrated that Nam elevated the intracellular cyclic adenosine 3',5'-monophosphate (cAMP) expression level by suppressing phosphodiesterase activity, leading to downstream cAMP-dependent protein kinase and cAMP-response element binding activation, thereby inducing the upregulation of SIRT1 (25). By conducting PH in mouse models, the results of this previous study were validated; Nam may promote the expression of SIRT1 in vivo. The factors leading to these conflicted observations may derive from cell type, dosage and exposure duration. In the present study, the results revealed that Nam at a dose of $250 \mathrm{mg} / \mathrm{kg}$ promoted the expression of SIRT1 and activated its deacetylating ability in residual liver tissue. Based on relevant literature, it was hypothesized that SIRT1 may be upregulated by Nam at a low 
dosage, and downregulated at a high dosage. However, since SIRT1-knocked-out mice could not be obtained, we were not able to clarify the specific mechanism under which Nam regulates the expression of SIRT1. Further studies are required to investigate the mechanism of how Nam modulates the expression of SIRT1 in regulating the metabolic response of the liver during regeneration.

In conclusion, the present study revealed that Nam may promote the proliferation of hepatocytes following $\mathrm{PH}$ and stimulate the activation of SIRT1 during liver regeneration. The present results provided insight into the potency of Nam in manipulating liver regeneration, which support a preclinical rationale to examine the clinical use of Nam for surgical resection recovery.

\section{Acknowledgements}

The authors thank the Core Facility of West China Hospital for their technical support.

\section{Funding}

The present study was supported by the Natural Science Foundation of China (grant nos. 81770615, 81700555, 81672882 and 81502441), the Science and Technology Support Program of Sichuan Province (grant no. 2017SZ0003), Tianqing Liver Diseases Research Fund of China Foundation for Hepatitis Prevention and Control (grant no. TQGB20170067) and Scientific Research Starting Foundation for Youths of Sichuan University (grant no. 2015SCU11999-9).

\section{Availability of data and materials}

The analyzed data sets generated during the study are available from the corresponding author on reasonable request.

\section{Authors' contributions}

H-FW, H-TL, J-XL and K-FY designed the experiments. H-FW, H-TL and LX performed the experiments. H-FW, M-HL and LL analyzed the data. H-FW, H-TL and K-FY wrote the manuscript. H-FW, K-FY and YZ revised the manuscript. YZ proposed the study concept and supervised the research. All authors reviewed the manuscript.

\section{Ethics approval and consent to participate}

Animal care and experimental protocols were in accordance with guidelines provided by the Institutional Animal Care and Use Committee of Sichuan University. Ethics approval was obtained from the Institutional Ethics Committee of Sichuan University.

\section{Patient consent for publication}

Not applicable.

\section{Competing interests}

The authors declare that they have no competing interests.

\section{References}

1. Ferlay J, Soerjomataram I, Dikshit R, Eser S, Mathers C, Rebelo M, Parkin DM, Forman D and Bray F: Cancer incidence and mortality worldwide: Sources, methods and major patterns in GLOBOCAN 2012. Int J Cancer 136: E359-E386, 2015.

2. Llovet JM, Di Bisceglie AM, Bruix J, Kramer BS, Lencioni R, Zhu AX, Sherman M, Schwartz M, Lotze M, Talwalkar J, et al: Design and endpoints of clinical trials in hepatocellular carcinoma. J Natl Cancer Inst 100: 698-711, 2008.

3. Llovet JM, Brú C and Bruix J: Prognosis of hepatocellular carcinoma: The BCLC staging classification. Semin Liver Dis 19: 329-338, 1999.

4. European Association For The Study Of The Liver; European Organisation For Research And Treatment Of Cancer: EASL-EORTC clinical practice guidelines: Management of hepatocellular carcinoma. J Hepatol 56: 908-943, 2012.

5. Bruix J and Sherman M; American Association for the Study of Liver Diseases: Management of hepatocellular carcinoma: An update. Hepatology 53: 1020-1022, 2011.

6. Lehmann K, Tschuor C, Rickenbacher A, Jang JH, Oberkofler CE, Tschopp O, Schultze SM, Raptis DA, Weber A, Graf R, et al: Liver failure after extended hepatectomy in mice is mediated by a p21-dependent barrier to liver regeneration. Gastroenterology 143: 1609-1619.e4, 2012.

7. Roayaie S, Jibara G, Tabrizian P, Park JW, Yang J, Yan L, Schwartz M, Han G, Izzo F, Chen M, et al: The role of hepatic resection in the treatment of hepatocellular cancer. Hepatology 62: 440-451, 2015.

8. Tremosini S, Reig M, de Lope CR, Forner A and Bruix J: Treatment of early hepatocellular carcinoma: Towards personalized therapy. Dig Liver Dis 42 (Suppl 3): S242-S248, 2010.

9. Clavien PA, Petrowsky H, DeOliveira ML and Graf R: Strategies for safer liver surgery and partial liver transplantation. N Eng J Med 356: 1545-1559, 2007.

10. Clavien PA, Oberkofler CE, Raptis DA, Lehmann K, Rickenbacher A and El-Badry AM: What is critical for liver surgery and partial liver transplantation: Size or quality? Hepatology 52: 715-729, 2010.

11. Chen AC and Damian DL: Nicotinamide and the skin. Australas J Dermatol 55: 169-175, 2014.

12. Oblong JE: The evolving role of the NAD+/nicotinamide metabolome in skin homeostasis, cellular bioenergetics, and aging. DNA Repair (Amst) 23: 59-63, 2014.

13. Stevens MJ, Li F, Drel VR, Abatan OI, Kim H, Burnett D, Larkin D and Obrosova IG: Nicotinamide reverses neurological and neurovascular deficits in streptozotocin diabetic rats. J Pharmacol Exp Ther 320: 458-464, 2007.

14. Kaneko S, Wang J, Kaneko M, Yiu G, Hurrell JM, Chitnis T, Khoury SJ and He Z: Protecting axonal degeneration by increasing nicotinamide adenine dinucleotide levels in experimental autoimmune encephalomyelitis models. J Neurosci 26: 9794-9804, 2006.

15. Thompson BC, Halliday GM and Damian DL: Nicotinamide enhances repair of arsenic and ultraviolet radiation-induced DNA damage in $\mathrm{HaCaT}$ keratinocytes and ex vivo human skin. PLoS One 10: e0117491, 2015.

16. Ashkani Esfahani S, Khoshneviszadeh M, Namazi MR, Noorafshan A, Geramizadeh B, Nadimi E and Razavipour ST: Topical nicotinamide improves tissue regeneration in excisional full-thickness skin wounds: A stereological and pathological study. Trauma Mon 20: e18193, 2015.

17. Gariani K, Ryu D, Menzies KJ, Yi HS, Stein S, Zhang H, Perino A, Lemos V, Katsyuba E, Jha P, et al: Inhibiting poly ADP-ribosylation increases fatty acid oxidation and protects against fatty liver disease. J Hepatol 66: 132-141, 2017.

18. Lehwald N, Tao GZ, Jang KY, Papandreou I, Liu B, Liu B, Pysz MA, Willmann JK, Knoefel WT, Denko NC and Sylvester KG: $\beta$-Catenin regulates hepatic mitochondrial function and energy balance in mice. Gastroenterology 143: 754-764, 2012.

19. Eriksson S, Prigge JR, Talago EA, Arnér ES and Schmidt EE: Dietary methionine can sustain cytosolic redox homeostasis in the mouse liver. Nat Commun 6: 6479, 2015.

20. Tummala KS, Gomes AL, Yilmaz M, Graña O, Bakiri L, Ruppen I, Ximénez-Embún P, Sheshappanavar V, Rodriguez-Justo M, Pisano DG, et al: Inhibition of de novo $\mathrm{NAD}(+)$ synthesis by oncogenic URI causes liver tumorigenesis through DNA damage. Cancer Cell 26: 826-839, 2014.

21. Imai S, Armstrong CM, Kaeberlein M and Guarente L: Transcriptional silencing and longevity protein Sir2 is an NAD-dependent histone deacetylase. Nature 403: 795-800, 2000. 
22. Timchenko NA,Harris TE, Wilde M,Bilyeu TA,Burgess-Beusse BL, Finegold MJ and Darlington GJ: CCAAT/enhancer binding protein alpha regulates $\mathrm{p} 21$ protein and hepatocyte proliferation in newborn mice. Mol Cell Biol 17: 7353-7361, 1997.

23. Lai HS, Chen WJ and Chen KM: Energy substrate for liver regeneration after partial hepatectomy in rats: Effects of glucose vs fat. JPEN J Parenter Enteral Nutr 16: 152-156, 1992.

24. Jin J, Iakova P, Jiang Y, Medrano EE and Timchenko NA: The reduction of SIRT1 in livers of old mice leads to impaired body homeostasis and to inhibition of liver proliferation. Hepatology 54: 989-998, 2011.

25. Li J, Dou X, Li S, Zhang X, Zeng Y and Song Z: Nicotinamide ameliorates palmitate-induced ER stress in hepatocytes via cAMP/PKA/CREB pathway-dependent Sirt1 upregulation. Biochim Biophys Acta 1853: 2929-2936, 2015.

26. Forbes SJ and Newsome PN: Liver regeneration-mechanisms and models to clinical application. Nat Rev Gastroenterol Hepatol 13: 473-485, 2016

27. Cardiff RD, Miller CH and Munn RJ: Manual hematoxylin and eosin staining of mouse tissue sections. Cold Spring Harb Protoc 2014: 655-658, 2014.

28. Ma S, Zhao Y, Sun J, Mu P and Deng Y: miR449a/SIRT1/PGC-1 $\alpha$ is necessary for mitochondrial biogenesis induced by T-2 toxin. Front Pharmacol 8: 954, 2018.

29. Wang S, Wang C, Turdi S, Richmond KL, Zhang Y and Ren J: ALDH2 protects against high fat diet-induced obesity cardiomyopathy and defective autophagy: Role of CaM kinase II, histone H3K9 methyltransferase SUV39H, Sirt1, and PGC-1a deacetylation. Int J Obes (Lond) 42: 1073-1087, 2018

30. Fausto N, Campbell JS and Riehle KJ: Liver regeneration. J Hepatol 57: 692-694, 2012.

31. Jin X, Zhang Z, Beer-Stolz D, Zimmers TA and Koniaris LG: Interleukin-6 inhibits oxidative injury and necrosis after extreme liver resection. Hepatology 46: 802-812, 2007.

32. Tajima T, Goda N, Fujiki N, Hishiki T, Nishiyama $Y$, Senoo-Matsuda N, Shimazu M, Soga T, Yoshimura Y, Johnson RS and Suematsu M: HIF-lalpha is necessary to support gluconeogenesis during liver regeneration. Biochem Biophys Res Commun 387: 789-794, 2009.

33. Bellet MM, Masri S, Astarita G, Sassone-Corsi P, Della Fazia MA and Servillo G: Histone deacetylase SIRT1 controls proliferation, circadian rhythm, and lipid metabolism during liver regeneration in mice. J Biol Chem 291: 23318-23329, 2016.

34. Jin J, Hong IH, Lewis K, Iakova P, Breaux M, Jiang Y, Sullivan E, Jawanmardi N, Timchenko L and Timchenko NA: Cooperation of C/EBP family proteins and chromatin remodeling proteins is essential for termination of liver regeneration. Hepatology 61: $315-325,2015$.

35. Rodgers JT, Lerin C, Haas W, Gygi SP, Spiegelman BM and Puigserver P: Nutrient control of glucose homeostasis through a complex of PGC-1alpha and SIRT1. Nature 434: 113-118, 2005.

36. Li X, Zhang S, Blander G, Tse JG, Krieger M and Guarente L: SIRT1 deacetylates and positively regulates the nuclear receptor LXR. Mol Cell 28: 91-106, 2007.
37. Longo CR, Patel VI, Shrikhande GV, Scali ST, Csizmadia E, Daniel S, Sun DW, Grey ST, Arvelo MB and Ferran C: A20 protects mice from lethal radical hepatectomy by promoting hepatocyte proliferation via a p21waf1-dependent mechanism. Hepatology 42: 156-164, 2005.

38. Okumura S, Teratani T, Fujimoto Y, Zhao X, Tsuruyama T, Masano Y, Kasahara N, Iida T, Yagi S, Uemura T, et al: Oral administration of polyamines ameliorates liver ischemia/reperfusion injury and promotes liver regeneration in rats. Liver Transpl 22: 1231-1244, 2016.

39. Hwang ES and Song SB: Nicotinamide is an inhibitor of SIRT1 in vitro, but can be a stimulator in cells. Cell Mol Life Sci 74: 3347-3362, 2017.

40. Weymann A, Hartman E, Gazit V, Wang C, Glauber M, Turmelle Y and Rudnick DA: p21 is required for dextrose-mediated inhibition of mouse liver regeneration. Hepatology 50: 207-215, 2009.

41. Brinkmann A, Katz N, Sasse D and Jungermann K: Increase of the gluconeogenic and decrease of the glycolytic capacity of rat liver with a change of the metabolic zonation after partial hepatectomy. Hoppe Seylers Z Physiol Chem 359: 1561-1571, 1978.

42. Delgado-Coello B, Briones-Orta MA, Macias-Silva M and Mas-Oliva J: Cholesterol: Recapitulation of its active role during liver regeneration. Liver Int 31: 1271-1284, 2011.

43. Asher G, Gatfield D, Stratmann M, Reinke H, Dibner C, Kreppel F, Mostoslavsky R, Alt FW and Schibler U: SIRT1 regulates circadian clock gene expression through PER2 deacetylation. Cell 134: 317-328, 2008

44. Chang HC and Guarente L: SIRT1 mediates central circadian control in the SCN by a mechanism that decays with aging. Cell 153: 1448-1460, 2013.

45. Nakahata Y, Kaluzova M, Grimaldi B, Sahar S, Hirayama J, Chen D, Guarente LP and Sassone-Corsi P: The NAD+-dependent deacetylase SIRT1 modulates CLOCK-mediated chromatin remodeling and circadian control. Cell 134: 329-340, 2008.

46. Sahar S and Sassone-Corsi P: Metabolism and cancer: The circadian clock connection. Nat Rev Cancer 9: 886-896, 2009.

47. Imai S and Guarente L: Ten years of NAD-dependent SIR2 family deacetylases: Implications for metabolic diseases. Trends Pharmacol Sci 31: 212-220, 2010.

48. Haigis MC and Sinclair DA: Mammalian sirtuins: Biological insights and disease relevance. Annu Rev Pathol 5: 253-295, 2010.

49. Jang SY, Kang HT and Hwang ES: Nicotinamide-induced mitophagy: Event mediated by high NAD+/NADH ratio and SIRT1 protein activation. J Biol Chem 287: 19304-19314, 2012.

50. Liu D, Gharavi R, Pitta M, Gleichmann M and Mattson MP: Nicotinamide prevents NAD+ depletion and protects neurons against excitotoxicity and cerebral ischemia: NAD+ consumption by SIRT1 may endanger energetically compromised neurons. Neuromolecular Med 11: 28-42, 2009. 